\title{
Underwater sound produced by individual drop impacts and rainfall
}

\author{
Pumphrey, Hugh C.; Crum, L. A.; Jensen, Leif Bjørnø
}

Published in:

Acoustical Society of America. Journal

Link to article, DOI:

10.1121/1.397353

Publication date:

1989

Document Version

Publisher's PDF, also known as Version of record

Link back to DTU Orbit

Citation (APA):

Pumphrey, H. C., Crum, L. A., \& Jensen, L. B. (1989). Underwater sound produced by individual drop impacts and rainfall. Acoustical Society of America. Journal, 85(4), 1518-1526. https://doi.org/10.1121/1.397353

\section{General rights}

Copyright and moral rights for the publications made accessible in the public portal are retained by the authors and/or other copyright owners and it is a condition of accessing publications that users recognise and abide by the legal requirements associated with these rights.

- Users may download and print one copy of any publication from the public portal for the purpose of private study or research.

- You may not further distribute the material or use it for any profit-making activity or commercial gain

- You may freely distribute the URL identifying the publication in the public portal

If you believe that this document breaches copyright please contact us providing details, and we will remove access to the work immediately and investigate your claim. 


\title{
Underwater sound produced by individual drop impacts and rainfall
}

\author{
Hugh C. Pumphrey and L. A. Crum \\ National Center for Physical Acoustics, University of Mississippi, P. O. Box 847. University, Mississippi 38677 \\ L. Bjørnø \\ Industrial Acoustics Laboratory, Technical University of Denmark, Building 425, DK-2800 Lyngby, \\ Denmark
}

(Received 1 June 1988; accepted for publication 28 November 1988)

\begin{abstract}
An experimental study of the underwater sound produced by water drop impacts on the surface is described. It is found that sound may be produced in two ways: first when the drop strikes the surface and, second, when a bubble is created in the water. The first process occurs for every drop; the second occurs for some impacts but not others. A range of conditions is described in which a bubble is produced for every drop impact, and it is shown that these conditions are likely to be met by a significant fraction of the raindrops in a typical shower. Underwater sound produced by artificial as well as real rain is reported. A comparison between artificial and real rain noise power spectra shows some deviations due to different drop-size distributions. Addition of surface tension reducing liquids to the water in the test tank caused a disappearance of the characteristic spectral peaks in the frequency range 14-16 $\mathrm{kHz}$. These peaks have been observed by several scientists during measurements of real rain. Our findings provide evidence for the theory that the 14- to $16-\mathrm{kHz}$ spectral peak is caused by the ringing of bubbles entrained in the water by the drop impact process.
\end{abstract}

PACS numbers: $43.30 . \mathrm{Nb}$

\section{HISTORICAL INTRODUCTION}

For nearly a century, scientists have studied what happens when a drop of water strikes a water surface. Worthington ${ }^{1}$ took flash photographs of the process in the 1890 s, while Mallock ${ }^{2}$ and $\mathrm{Bragg}^{3}$ put forward several theories in an attempt to describe the sound produced. These theories were mostly based on the resonance of an open-ended cavity at the water surface, and are now known to be erroneous.

Bragg's work, which also discussed the sound produced by running water, lead Minnaert ${ }^{4}$ to study the sound radiated by an oscillating bubble that was released into a tank of water. He also derived a formula for the resonance frequency of a gas bubble in a liquid, which is still regarded as a good approximation. Minnaert must have suspected that his work was related to drop impacts, for his paper ends with these words: "It remains to investigate ... if the sounds of falling drops cannot have the same origin as the bubble sounds."

The first thorough investigation of the sounds produced by drop impacts was made by Franz $z^{5}$ in 1959; this article has been the standard reference on the subject ever since. Franz used high-speed movie photography to show how the water behaved during the impact process. At the same time, he recorded the sound generated in the water and was thus able to show which features of the sound trace were associated with each phase of the impact. The main sources of underwater sound from a splash that he discovered were: (1) the impact and passage of the body (water droplet) through the free water surface leading to the establishment of flow; (2) resonance vibrations of the body, if it has rigidity; and (3) volume pulsations of bubbles of air in the water. The initial impact sound was a sharp pulse, while the bubble sound was a decaying sinusoid. (The body vibrations will not concern us in this article as a drop of water is not rigid.) Franz observed that bubbles were not produced by every drop and that their occurrence was more or less random.

Franz found that the sound pressure radiated by the initial entry of the droplet increases systematically with increases in droplet size and impact velocity, and that the halfoctave spectra of the impact sound in water show a broad maximum in the frequency range between 1 and $10 \mathrm{kHz}$. He also showed that drop impacts seem to behave as dipole sources with vertical axes, as one would expect for a simple source near a free surface.

In addition to his work on single drops, Franz studied the sound produced by a spray of water. He attempted to predict the acoustic power spectrum of rain from his results, suggesting very broad, flat spectra, which peak at $3 \mathrm{kHz}$. His predictions do not agree well with more recent field measurements.

One of the first attempts to describe the underwater noise spectra produced by real rainfall was published by Heindsmann et al., ${ }^{6}$ who found that, during the heaviest rainfall, the sound-pressure spectrum level was approximately constant at $77 \mathrm{~dB} r e: 1 \mu \mathrm{Pa}$ from below $1 \mathrm{kHz}$ to above $10 \mathrm{kHz}$.

A study of underwater noise due to precipitation on the surface of a small, shallow lake was published by $\mathrm{Bom}^{7}$ for the frequency band $0.3-9.6 \mathrm{kHz}$. He found that the noise level in $\mathrm{dB}$ versus the logarithm of the rain rate in the interval $1-25 \mathrm{~mm} / \mathrm{h}$ can be represented by a straight line. A comparison with Franz's predictions shows a significant difference in absolute level, in particular at low frequencies; however, Bom's underwater noise spectrum levels seem to 
peak at 3-4 kHz for several rain rates, as Franz predicted.

Scrimger ${ }^{8}$ recorded the power spectrum of natural rain falling onto a lake. He observed a feature that had gone unnoticed by previous authors: a spectral peak at about $15 \mathrm{kHz}$, with a steep slope on the low-frequency side. Nystuen ${ }^{9,10}$ reported a similar result and developed a theory to explain it. He used a numerical model for the drop splash flow field that allows the study of multiple free surfaces to be performed and that permits variation in surface tension, viscosity, and droplet shape to be introduced. This enabled him to predict the shape of the initial short, high-amplitude acoustic pulse and the nonacoustic dynamic pressure that is associated with the flow field. He also discussed Franz's description of the initial impact pulse, which disagreed with his own. Nystuen explained the 14- to $16-\mathrm{kHz}$ peak that he observed in the spectrum of rainfall in terms of the initial impact sound alone, ignoring bubbles on the basis that not every drop produces them.

Scrimger et al. " made an extensive study of the spectral characteristics of underwater noise generated by rain falling onto the surface of a freshwater lake. Their measurements were made using a bottom-mounted hydrophone at a depth of $35 \mathrm{~m}$. For wind speeds less than $1.2 \mathrm{~m} / \mathrm{s}$, the authors found the rain noise spectra to have a sharp peak at $13.5 \mathrm{kHz}$ with a 9-dB/oct falloff on the high-frequency side and a 60$\mathrm{dB} /$ oct falloff on the low-frequency side. These results confirmed the presence of the 14- to $16-\mathrm{kHz}$ peak observed by Nystuen. These spectra also showed many features in agreement with Bom's data between 2 and $10 \mathrm{kHz}$.

The apparent lack of agreement that exists between the rain noise spectra measured and calculated by the authors $^{5-11}$ calls for a more systematic and comprehensive study of the mechanisms leading to rain-produced noise in the sea and to the correlation between these mechanisms and some characteristic features of the underwater noise spectra produced by rain. This article describes a thorough investigation of the sound produced by single-drop impacts, which confirms and extends some of Franz's findings. It presents the acoustic spectra of both artificially and naturally produced rainfall and explains some spectral characteristics in terms of bubble sounds. The studies reported have been performed in collaboration between a U. S. based group at the National Center for Physical Acoustics (NCPA), Oxford, MS and a Danish group at the Technical University of Denmark (TUD). The single-drop impact studies have mostly been made by the NCPA group, while the larger part of the artificial and real rain studies have been performed by the TUD group.

\section{EXPERIMENTAL PROCEDURE}

In all studies, underwater sound was received with a hydrophone ( $B \& K$ 8103). While the NCPA group used a charge amplifier (B\&K 2635) connected to a LeCroy 9400 digital oscilloscope for the pressure signal monitoring, the TUD group used a dual channel FFT analyzer ( $B \&$ K 2032) for the signal processing. For single-drop impacts, the most important method of study used by the NCPA group was high-speed movie photography. The camera was a Photec IV rotating prism instrument, which was generally run at 1000,2000 , or 3000 frames per second. The splashes were filmed against a bright, diffusely illuminated background. The amplified sound signal was passed to an oscilloscope with the time base switched off. The oscilloscope screen was photographed directly onto the high-speed movie film via an auxiliary lens on the camera. The result of this procedure is a single film with a sequence of photographs of the splash on one edge and a continuous record of the sound pressure down the other. Figure 1 shows a schematic of this apparatus.

Further careful and systematic studies were made to discover how the sound of a single-drop impact varies with such parameters as impact velocity, drop size, and surface tension. Drop size was controlled by allowing the drops to fall from different sized hypodermic needles, whose ends were ground square. This method fails for drops whose diameters are smaller than about $2 \mathrm{~mm}$. These smaller drops were produced by drawing the required volume of water into a microliter syringe, forcing it out to form a drop, and then persuading it to fall by tapping the syringe. The magnitude of the impact velocity $v_{I}$ was controlled by allowing the drops to

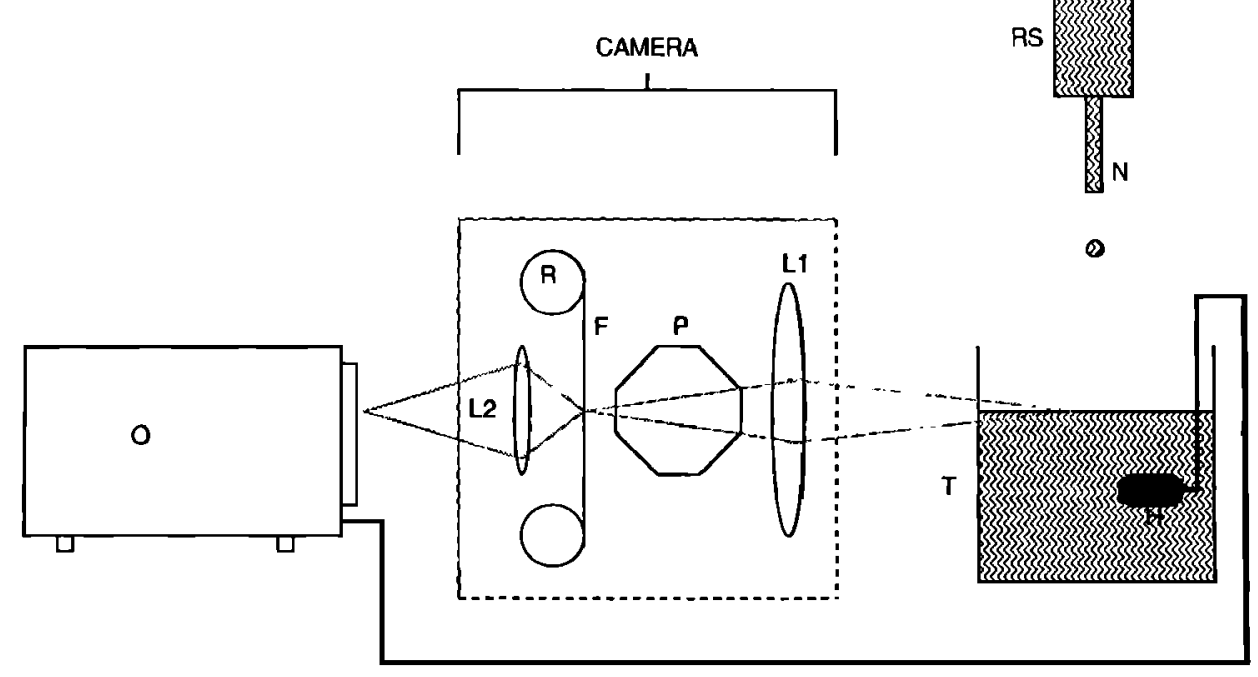

FIG. 1. System used to pholograph drop impacts. Water from the reservoir RS falls from the needle $\mathrm{N}$ into the glass tank $\mathrm{T}$. Its image is focused onto the film $F$ by the lens L1. The film moves continuously between the reels $R$; the image moves along with the film because it passes through the rotating prism $P$. The sound is detected by the hydrophone $H$ and displayed on the oscilloscope $O$. The time base of the oscilloscope is switched off so that the spot is deflected in a plane perpendicular to the paper, and its image is focused onto the moving film by lens $\mathrm{L} 2$ to give a continuous trace on the film. 
fall from different heights and was calculated from the following equation ${ }^{12}$ :

$$
v_{I}=v_{T}\left[1-\exp \left(-2 g h / v_{T}^{2}\right)\right]^{1 / 2} .
$$

In this equation, $h$ is the height of fall and $g$ is the magnitude of the acceleration due to gravity. The terminal velocity $v_{T}$ is a function of the drop diameter $d$ and was found from $d$ by a polynomial fit to some standard data. ${ }^{13,14}$ Surface tension was adjusted by adding a surfactant to the water; Kodak Photoflo was used by the NCPA group. The TUD group used a sulfo detergent whose brand name is Nuren Opvask and whose active ingredient has the trade name Syndeter.

Real and artificial rain were both used to study the sound produced by multiple-drop impacts. The artificial rain was produced at TUD by two different systems. The first was a shower head with a random distribution of hypodermic needles, the diameters of which were chosen so that the drop-size distribution produced was similar to that of real rain. The distribution was checked with a distrometer and was good for drop diameters greater than $2 \mathrm{~mm}$. This first system was quite similar to the shower used by Franz. The second system consisted of a coiled up water hose with a number of holes of various sizes drilled through the hose wall. Jets of water from these holes rose into the air and fell onto the free surface of a water-filled tank of the dimensions $2.5 \times 1 \times 1 \mathrm{~m}$. The hydrophones could, by the use of an adapter, be positioned at various water depths with an accuracy of about $\pm 0.5 \mathrm{~mm}$. The temperature of the water in the tank varied from 2 to $21^{\circ} \mathrm{C}$, thus representing winter as well as summer conditions. $\mathrm{A}$ similar system to the second one described above was used at NCPA, where the tank had dimensions $1 \times 1 \times 1 \mathrm{~m}$.

The TUD tank facility was used for real-rain studies when the tank was placed in a parking lot free from turbulence, etc., created by buildings. Measurements of the angle

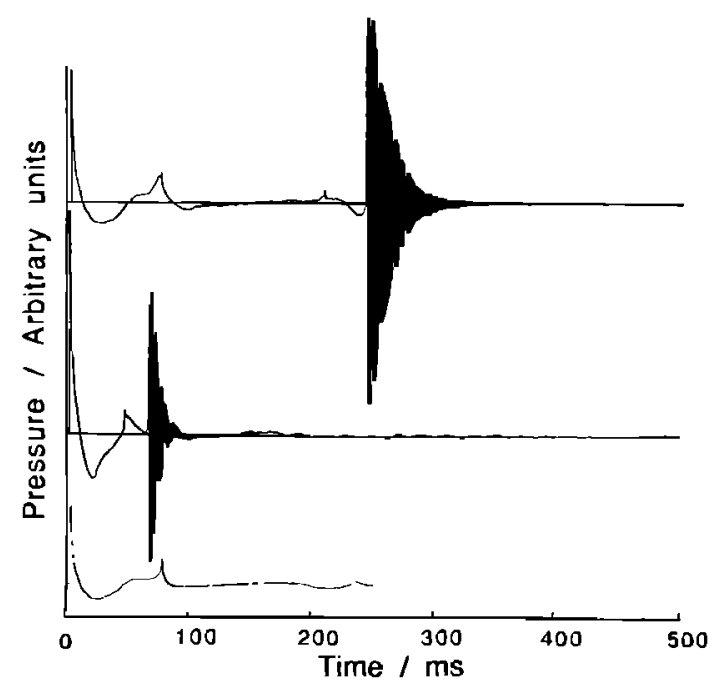

FIG. 2. Irregular entrainment. Sounds produced by drops of $5.2-\mathrm{mm}$ diameter impacting at a velocity of about $6.8 \mathrm{~m} / \mathrm{s}$ after falling from a height of 3.5 $\mathrm{m}$. The top trace shows a bubble sound at a time of $250 \mathrm{~ms}$; the center trace shows one at a time of $60 \mathrm{~ms}$. The lower trace shows no bubble sound, but only the initial impact and the nearfield hydrodynamic pressure variations. The bubble sounds appear to have a longer duration than predicted by theory, but this is only due to reflections from the tank walls (data from the TUD group; note that the units of pressure are different for each trace). of incidence of the real rain was done using a device permitting an accuracy of $\pm 2^{\circ}$. The artificial and real-rain noise signals were stored on floppy disks using a HP 300 series computer, which permits a comparison of several spectra. A few recordings of rain noise were done using an HP 3585A spectrum analyzer in order to investigate if spectral characteristics were to be found in the frequency range above 25.6 $\mathrm{kHz}$, which is the limit for the application of the B\&K 2032 analyzer.

\section{RESULTS}

\section{A. Single drops}

\section{General observations}

Figures 2 and 3 show typical pressure-time traces produced by single-drop impacts; the two types of sound oberved by Franz can be seen clearly. As shown in Fig. 2, one always observes an initial pulse, but the occurrence of bubble sounds is not always predictable. For identical drops, it may occur at various times after the drop impact, or not at all. We shall refer to this process as irregular entrainment. The decaying sinusoidal waveforms show great variation in freuency from one impact to another, indicating that bubbles produced in this way may be very large or very small.

Most of the low-frequency pressure variations that occur after the initial impact are nearfield hydrodynamic effects. The sharp peak that occurs at a time of $70 \mathrm{~ms}$ was caused by the impact of a small drop (Plateau's spherule) that detached from the hypodermic needle at the same time as the main drop and followed it down. The slight variation in pressure that occurs about $200 \mathrm{~ms}$ after the initial impact is caused by the collapse and reimpacting of a water column and several small drops that are thrown up by the splash.

Figure 3 shows an additional bubble entrainment mechanism that Franz did not discover because all of the drops he

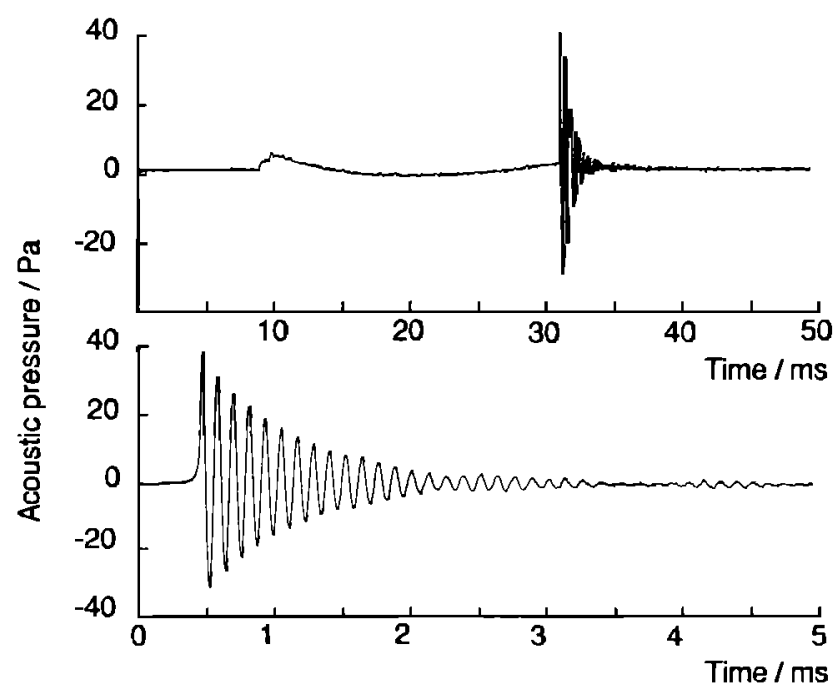

FIG. 3. Regular entrainment. Sounds produced by drops of 3.0-mm diameter impacting at a velocity of $2.0 \mathrm{~m} / \mathrm{s}$. The upper trace shows the whole process, with the initial impact occurring at a time of about $8 \mathrm{~ms}$ and the bubble sound at $32 \mathrm{~ms}$. The lower trace is an expansion along the time axis of a part of the upper one and shows the bubble sound in greater detail (data from the NCPA group). 
studied were too large or had too great an impact velocity. Drops of certain sizes, impacting with certain velocities, will produce a bubble every time. The bubbles produced are usually all of similar sizes. We shall call this process regular entrainment.

Figures 4-6 are sequences of selected frames from highspeed movies. The frames are in order but are not necessarily
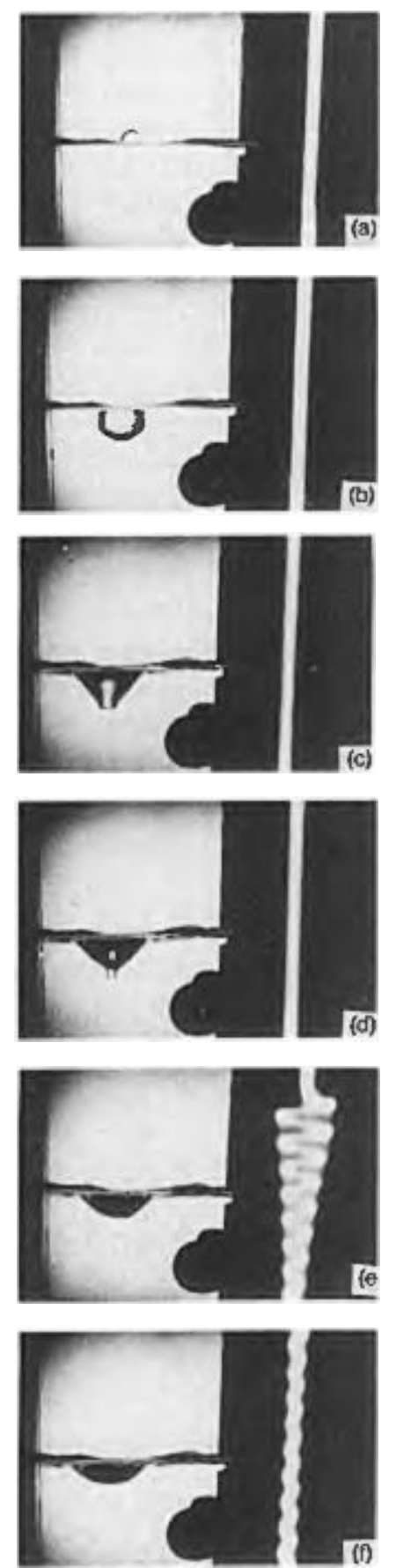

4. Regular entrainment. Frames from a high-speed movie film showing a drop of $3.8-\mathrm{mm}$ diameter impacting at $1.5 \mathrm{~m} / \mathrm{s}$. Total time is about $32 \mathrm{~ms}$.

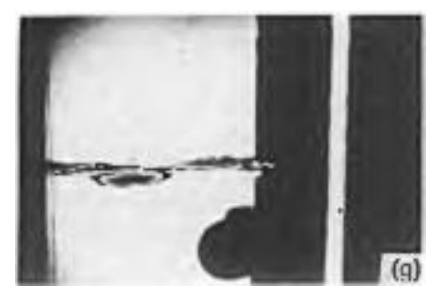

sequential. Figure 4 shows regular entrainment. The initial impact sound occurs in frame 4(a) but is too quiet to be seen above the background noise. The bubble sound is easily observed in frames $4(\mathrm{e})$ and (f); it begins just at the moment when the bubble detaches.

Figure 5 was taken under identical conditions to Fig. 4, except that the surface tension was lowered to about $30 \mathrm{dyn} /$
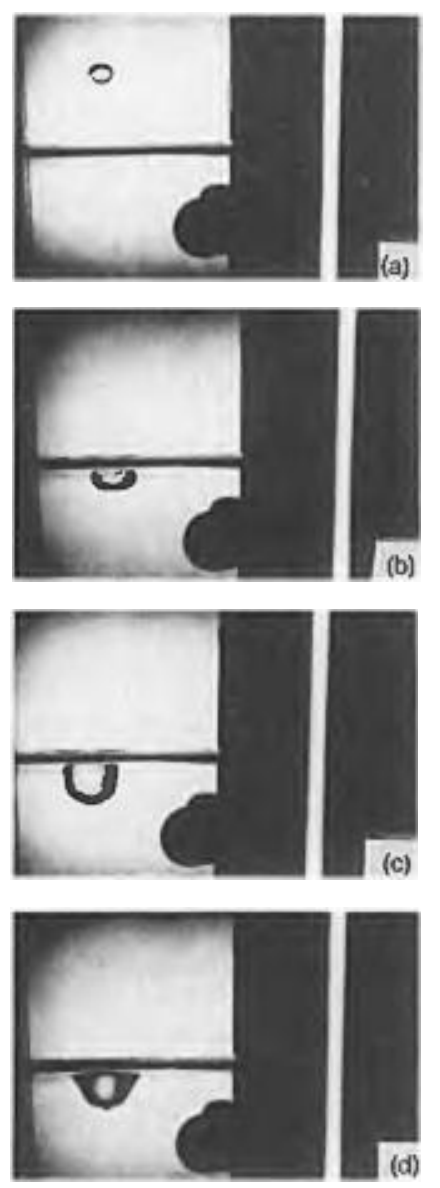

FIG. 5. As in Fig. 4, bul with surface lension level lowered from 72 dyn $/ \mathrm{cm}$ lo aboul $30 \mathrm{dyn} / \mathrm{cm}$.
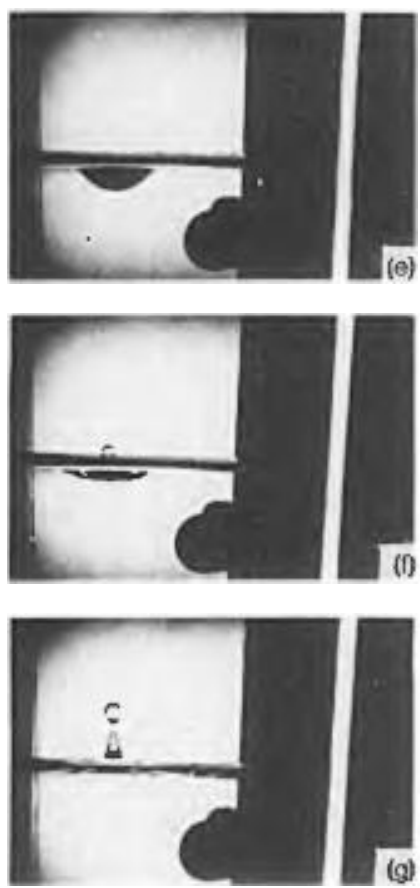
$\mathrm{cm}$ by the addition of a surfactant. The major difference here is that no bubble is entrained, and, consequently, the typical damped sine wave sound is not observed.

Figure 6 shows a larger drop impacting at a greater speed. The initial impact sound can be seen clearly in frame 6(a), and bubbles are formed at several stages in the process: see frames $6(\mathrm{e})$ and $(\mathrm{g})$. The bubble in frame 6(e) was trapped as a result of the impact of a small drop that followed
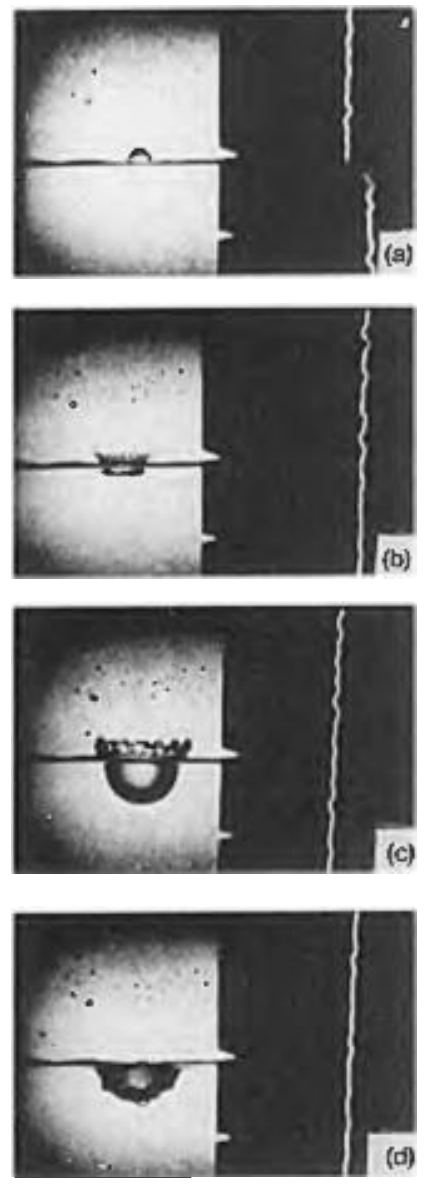

FIG. 6. Irregular entrainment. A drop of 5.8-mm diameler impacting at $2.4 \mathrm{~m} / \mathrm{s}$. Note that the bubbles formed in frames $(\mathrm{e})$ and $(\mathrm{g})$ are very small and the frequency of the sound is consequently very high. Total time is about $200 \mathrm{~ms}$ the main drop down. Franz $z^{5}$ and others ${ }^{15}$ have reported this phenomenon. The bubble in Fig. $6(\mathrm{~g})$ is trapped by the reimpacting drop seen in Fig. 6(f).

\section{Initial impact pulses}

Figure 2 shows the initial impact pulse to consist of two distinct parts. The first is a sharp leading edge, which is true radiated sound and which lasts for only $10-40 \mu \mathrm{s}$. The pressure variation that follows the leading edge and that lasts for $30-70 \mathrm{~ms}$ is a nearfield hydrodynamic effect related to the flow established on and near the impact site. This effect is not true sound at all and will be noticed only in the vicinity of the impact site. The true initial impact pressure $p_{l}$ is proportional to the drop diameter to the power $2.1 \pm 0.6$. Figure 7 shows that $p_{I}$ is also proportional to the impact velocity $v_{I}$ to a power between 2.5 and 3. This result is supported by Franz's work, in which he wrote the dimensionless impact pressure in a form which suggests that $p_{I} \propto v_{I} .{ }^{3}$ It does not agree with the theory suggested by Nystuen, ${ }^{9,10}$ which assumes the initial impact pulse to be a water hammer effect. This gives $p_{I} \propto \rho c v_{I}$, where $\rho$ is the liquid density and $c$ is the velocity of sound. The initial impact pulse is not sensitive to changes in surface tension.

\section{Bubble sounds}

The damped sine wave that is seen in Figs. 2-4 must be connected intimately with the bubble which is released at the moment that the oscillation begins. The most likely mechanism appeared to be radial oscillations of the bubble itself, and several experiments were done to verify this theory. Minnaert' $\mathrm{s}^{4}$ equation for the resonance frequency $f$ of a bubble is

$$
f=(\pi d)^{1}\left(3 \kappa P_{0} / \rho\right)^{1 / 2} .
$$

where $d$ is the bubble diameter, $P_{o}$ is the static pressure at the bubble, $\rho$ is the density of water, and $\kappa$ is the polytropic exponent of the gas in the bubble. The oscillation frequency and bubble diameter were measured directly from the film, and the equation was checked by plotting $f$ against $1 / d$. The agreement was reasonably good.

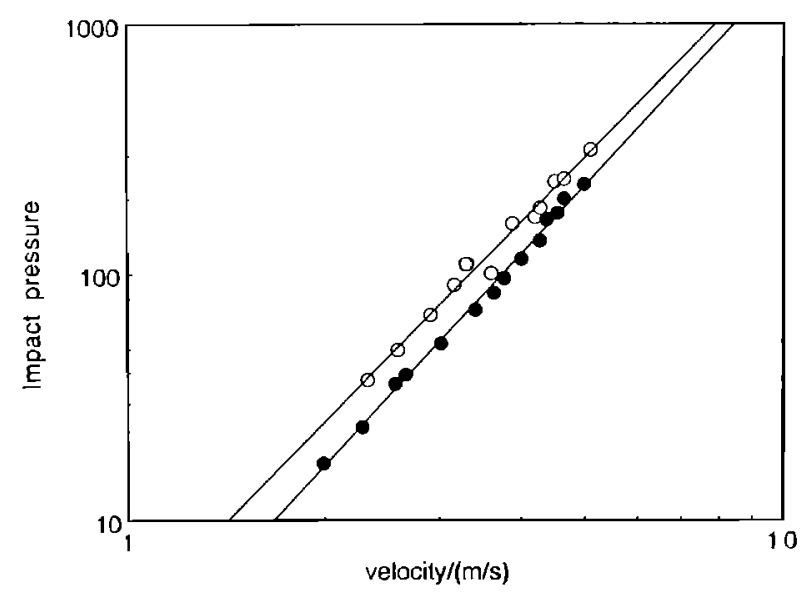

FIG. 7. Logarithmic plot of peak acoustic pressure from initial impact versus drop impact velocity. Data are shown for two drop diameters: $3.8 \mathrm{~mm}$ (open circles) and $2.5 \mathrm{~mm}$ (closed circles). The best fit lines show power laws of 2.6 and 2.83 , respectively. 
In addition, the damping constant $\beta$ was measured for a large number of bubbles. The pressure, as a function of time, can be approximately represented by

$$
p=p_{0} e^{-\beta t} \sin 2 \pi f t \text {. }
$$

The amplitude at each half-cycle is therefore given by

$$
p=p_{0} e^{-i n / f},
$$

where $n=0,1 / 2,1,3 / 2 \ldots$. We plotted $\log p$ against $n$ to obtain a straight line with slope $-\beta / f$. The results obtained agree fairly well with theoretical predictions; we intend to present them in a subsequent article.

\section{Conditions for bubble production}

The two sorts of bubble entrainment, regular and irregular, have been defined previously. A careful study was made of the heights of fall and drop sizes necessary for regular entrainment to occur, that is, for every drop of that size and impact velocity to produce a bubble. The results are presented in Fig. 8(a). This is a graph with drop diameter along the horizontal axis and drop impact velocity on the vertical axis.

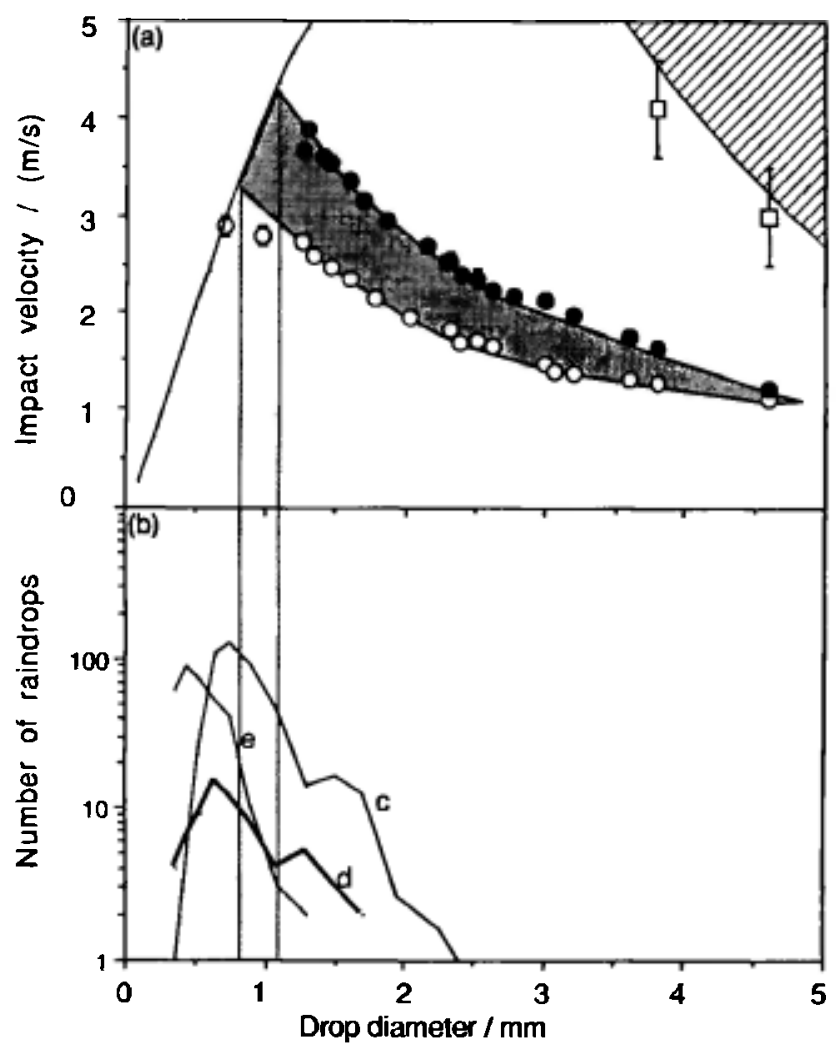

FIG. 8. (a) Conditions necessary for bubble entrainment. Regular entrainment occurs in the shaded region in the center of the figure, irregular entrainment occurs (very approximately) in the striped region at the top of the graph. Most of the data points used to draw the boundary of this region are off the edge of the graph. The line at the left is the terminal velocity curve for raindrops, and the two vertical lines are at the drop diameters at which the terminal velocity curve passes through the regular entrainment region, i.e., 0.8 and $1.1 \mathrm{~mm}$. (b) Drop-size distributions for three different rain showers. This shows that a large proportion of the drops in a typical rain shower are in the size range for which the terminal velocity curve intersects the shaded region, i.e., between the two vertical lines. Hence, many raindrops fulfill the conditions for regular entrainment. The units on the ordinate are: number of drops in a $0.1-\mathrm{mm}$ size range striking an area of $50 \mathrm{~cm}^{2}$ in a time of $90 \mathrm{~s}$ [data taken from J. A. Scrimger et al. "1].
Any drop impact can be represented by a point in this plane. The shaded area represents impacts that will cause regular entrainment; its boundaries are cuves drawn through experimental points. The area below this region represents drops that impact too slowly to cause regular entrainment; the area above represents drops that impact too fast. The striped area at the top right-hand corner shows approximately the region where irregular entrainment occurs.

In the left of the figure is the terminal velocity curve for drops-all raindrop impacts are assumed to lie on this curve because the drops have all fallen from a great height. We can expect raindrops in the size range $0.8-1.1 \mathrm{~mm}$ to produce a bubble at every impact because the terminal velocity curve lies within the regular entrainment region for these sizes. Furthermore, as Fig. 8(b) shows, a large proportion of raindrops lie in this size range. This enables us to formulate a theory of the sound produced by rain in terms of the sound emitted by regularly entrained bubbles.

Finally, we note the effect of surface tension on regular entrainment. This is difficult to measure exactly, but it is clear that, at surface tensions of $48 \mathrm{dyn} / \mathrm{cm}$ or below, the process does not occur at all. This result is demonstrated in Fig. 5.

\section{B. Artificial and real rain}

The acoustic power spectrum of rain has been described most thoroughly by Scrimger et al., " and the main features have been confirmed by Nystuen. ${ }^{9.10}$ Typical examples measured by the several groups are shown in Fig. 9. The most obvious feature is the peak at about $14-16 \mathrm{kHz}$ with a steep falloff on the low-frequency side. Comparison with data on drop size suggests that this peak is associated with drops whose diameters are below $1.2 \mathrm{~mm}$. Larger drops are most probably correlated with the low-frequency part of the spectra in Fig. 9.

The sound of artificial rain produced by the shower head is compared with the real rain noise spectrum in Fig. 10. This figure shows a considerable deviation between the two spectra. The main reason is probably that it is extremely

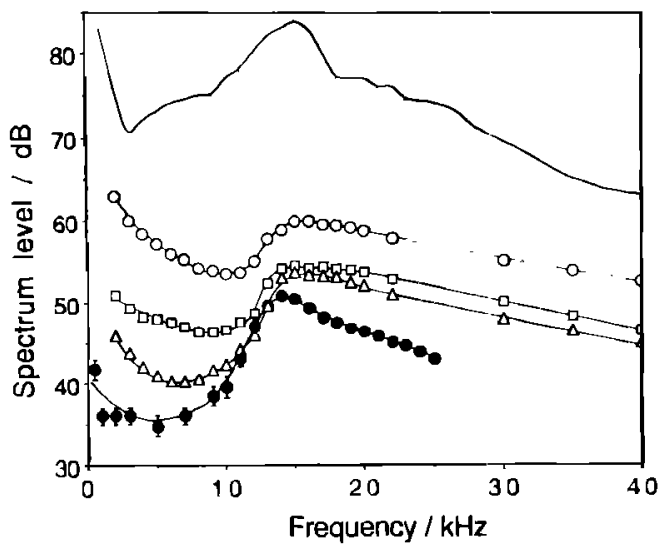

FIG. 9. Acoustic power spectra produced by rain of different drop-size distributions. Continuous line: very heavy rain [data from Nystuen'] points, light rain with the drop-size distributions shown in Fig. 8(b). circles: c; squares: d; triangles: e [data from J. A. Scrimger et al." ]; closed circles: light rain [data from TUD]. All spectrum levels are in dB re: 1 $\mu \mathrm{Pa}^{2} / \mathrm{Hz}$, except the TUD set whose reference level is arbitrary. 


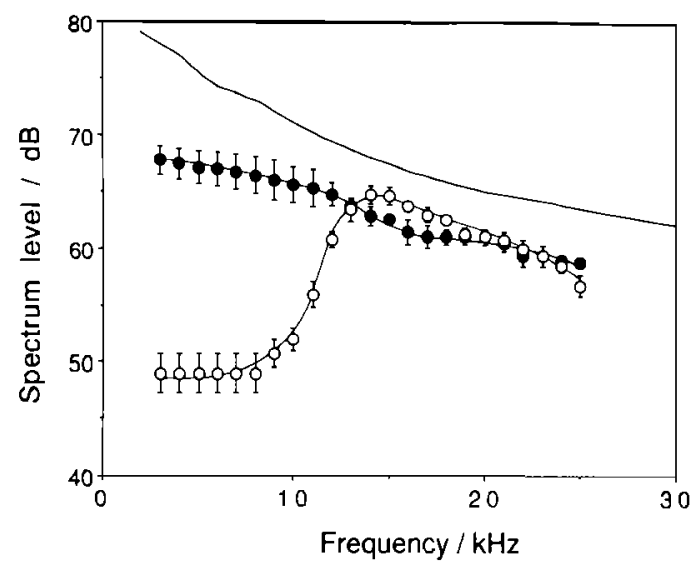

FIG. 10. Acoustic power spectra of real rain (open circles) and the TUD shower (closed circles). The line is the spectrum obtained by Franz from his shower. This has a different reference level, but its shape is similar to that from the TUD shower, neither being like that from real rain. Here, $\mathrm{dB}$ reference level is $1 \mu \mathrm{Pa}$ for Franz's data and arbitrary for the TUD data.

difficult to produce single drops having diameters between $1.5-2 \mathrm{~mm}$ without oscillating the hypodermic needle. Most drops from the shower head have diameters greater than 2 $\mathrm{mm}$, thus falling outside the regular entrainment region's drop sizes. The spectrum obtained by Franz for his shower is shown for comparison. We can see that the spectra from both shower systems have a similar shape, which is not at all like that of real rain.

The underwater sounds of artificial rain produced by small holes in a water hose at TUD and by a similar system at NCPA are compared with a real-rain noise power spectrum in Fig. 11. These systems produce a greater number of small droplets in the regular entrainment range of drop sizes, and therefore their spectra are quite similar to that of real rain. The artificial rain produces more low-frequency sound than the real rain; this low-frequency sound may be due to irregular entrainment of large bubbles. A characteristic feature of Fig. 11 is the spectral peak position for the artificial rain; it is $1-1.5 \mathrm{kHz}$ higher than the real-rain spectral peak. These differences are probably caused by some deviations in the drop-size distribution between the artificial and the real rain which emphasize the difficulties connected with reproducing nature.

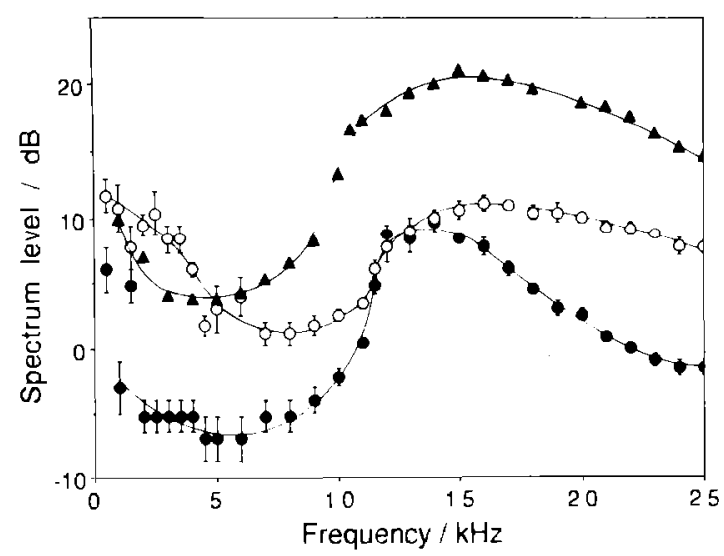

FIG. 11. Acoustic power spectra of real rain (closed circles), TUD artificial rain (open circles), and NCPA artificial rain (triangles). Here dB reference levels are arbitrary.

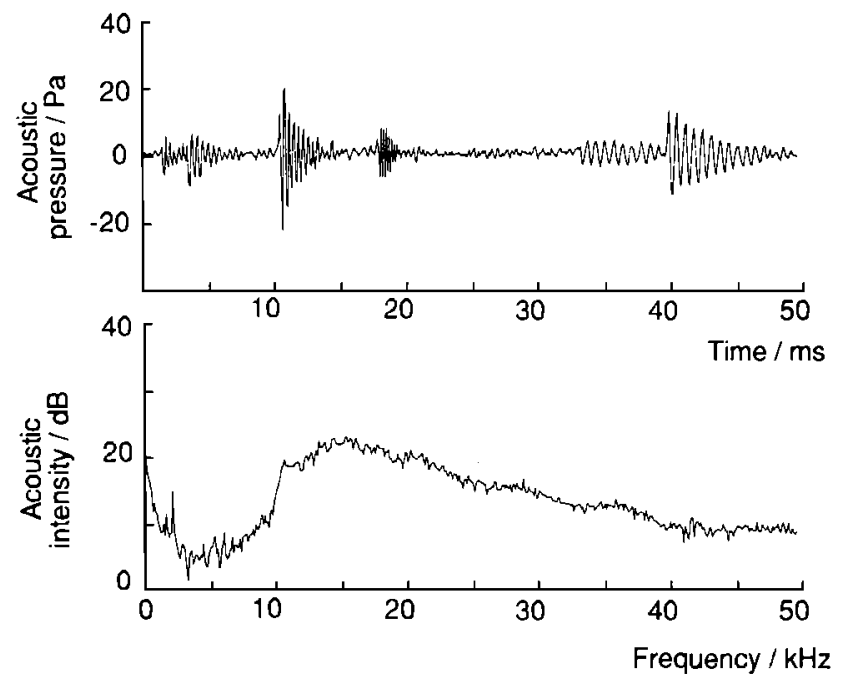

FIG. 12. A sound-pressure trace produced by a spray of water falling onto a large tank of water (above) and an averaged power spectrum of 200 such traces (below). Here $\mathrm{dB}$ reference level is arbitrary.

Figure 12 shows an artificial rain spectrum recorded at NCPA and a typical oscilloscope trace, which is mainly composed of bubblelike oscillations. In order to observe these individual oscillations, the hydrophone must be very close to the water surface. As it is moved deeper, the power spectrum does not change, but the individual bubble traces run together and become unrecognizable. This effect occurs because the hydrophone is averaging over a larger area and, therefore, more drop impacts. The drop-size distribution was estimated by catching drops and measuring their volumes with a microliter syringe. Impact velocities were calculated from Eq. (1). The smallest drops had diameters of about $0.7 \mathrm{~mm}$ and velocities of about $2.7 \mathrm{~m} / \mathrm{s}$, while the largest drops had diameters of approximately $1.5 \mathrm{~mm}$ and velocities in the range $3-4 \mathrm{~m} / \mathrm{s}$. This means that most of the drops fell between the regular entrainment limits of Fig. 8, which suggests that the main peak in both real and artificial rain is caused by regular entrainment of bubbles.

Single-drop experiments show that adding detergents to the water has a large effect on the entrainment of bubbles. This suggested that it would be instructive to study the ef-

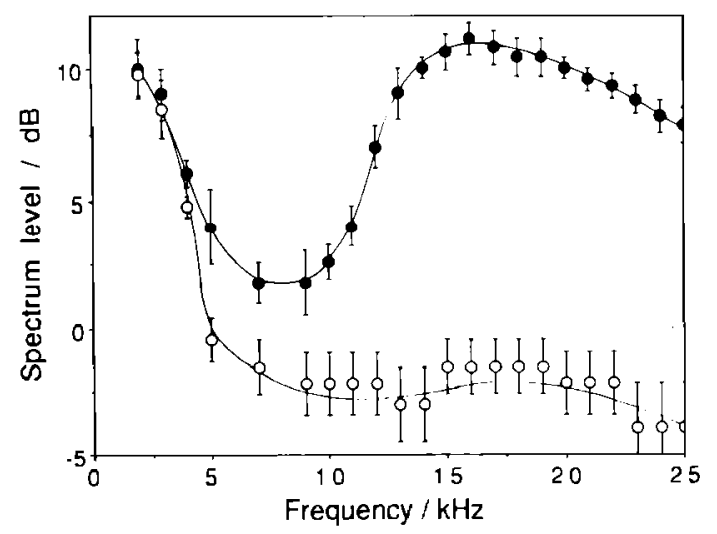

FIG. 13. Acoustic power spectra of TUD artificial rain falling into clean water (closed circles) and water with sulfo detergent added (open circles). Here, $\mathrm{dB}$ reference level is arbitrary. 
fects of surfactants on the sounds produced by rain. Figure 13 shows the power spectrum produced by artificial rain before and after the addition of $0.1 \mathrm{ppm}$ of sulfo to the water in the test tank, lowering the surface tension to $32 \mathrm{dyn} / \mathrm{cm}$. While the low-frequency part of the spectrum is unchanged, the spectral peak around $15.6 \mathrm{kHz}$ totally disappears.

This experiment was repeated with real rain; Fig. 14 shows the sound power spectrum before and after adding 0.1 ppm of sulfo to the water in the tank. Again, the low-frequency part of the spectrum is little changed, but the characteristic spectral peak around $14-16 \mathrm{kHz}$ has totally disappeared. The measurements in Figs. 13 and 14 were all done with the hydrophone at a depth of $0.2 \mathrm{~m}$.

The NCPA group examined the effect that the addition of Kodak Photoflo had on the sound produced by artificial rain. The decreasing surface tension leads to a decrease in amplitude of the spectral peak around $14-16 \mathrm{kHz}$, and to a total elimination of the peak at a surface tension of about 48 dyn/cm, as shown in Fig. 15. This is approximately the value required to eliminate regular entrainment.

\section{DISCUSSION}

The results of these experiments show that an impacting water drop can cause underwater sound by two separate mechanisms. First, there is the initial impact sound, which occurs for every impact. Second, there is the bubble oscillation, which, when it occurs, is a stronger acoustic source than the initial impact, but does not occur for every drop. It is also found that, for certain drop sizes and impact velocities, the bubble sound occurs for every impact. An extrapolation of the results suggests that this regular entrainment will occur for raindrops at their terminal velocity provided those drops are in a certain range of sizes. Specifically, their diameters should be between 0.8 and $1.1 \mathrm{~mm}$. Drops of these sizes are common in most types of rain, especially light showers [see Fig. 8(b)], so we can expect rain to produce large numbers of regularly entrained bubbles.

Scrimger et al. ${ }^{11}$ and Nystuen' ${ }^{9,10}$ field measurements seem to show a correlation between the $14-$ to $16-\mathrm{kHz}$ peak

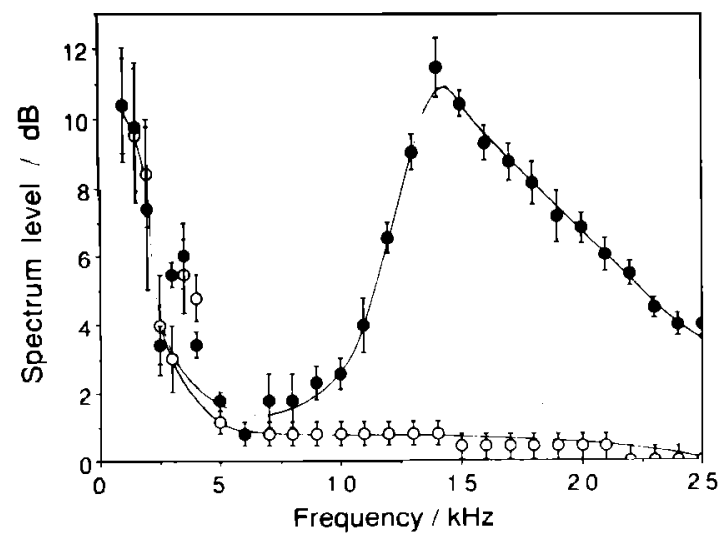

FIG. 14. Acoustic power spectra of real rain falling into clean water (closed circles) and water with sulfo detergent added (open circles). Here, dB reference level is arbitrary.

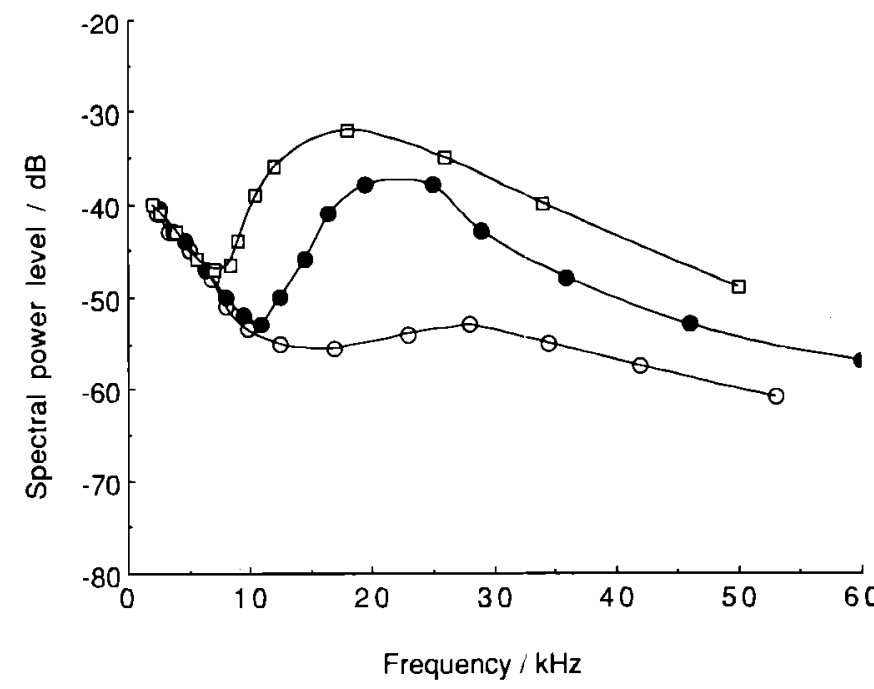

FIG. 15. Acoustic power spectra of a spray of water falling onto a large tank of water at various surface tensions. Open squares: $72 \mathrm{dyn} / \mathrm{cm}$ (clean water ); closed circles: $61 \mathrm{dyn} / \mathrm{cm}$; open circles: $48 \mathrm{dyn} / \mathrm{cm}$. The $\mathrm{dB}$ reference level is arbitrary.

and small raindrops and also between sound at lower frequencies and large raindrops. The comparison between the spectra c, d, and e in Fig. 9, as defined in the caption, and the corresponding rainfall data in Fig. 8(b) is a typical example. At a frequency of $15 \mathrm{kHz}, \mathrm{d}$ and e have the same spectral level, whereas, at $5 \mathrm{kHz}, d$ is much louder. This suggests that they should have similar numbers of drops in the 0.8- to 1.1$\mathrm{mm}$ range of sizes and that $\mathrm{d}$ should have more large drops. Figure 8(b) shows that this is, indeed, the case. Shower $c$ has more drops than $\mathrm{d}$ or $\mathrm{e}$ for all drop diameters above $0.6 \mathrm{~mm}$, and its spectrum is, therefore, louder than $d$ or $e$ at all frequencies. Shower e has the most drops with diameters less than $0.6 \mathrm{~mm}$ and yet has the quietist spectrum. It, therefore, seems likely that these very small drops have little effect on the sound produced. This is only a small amount of data, but the results of Nystuen and the TUD group are compatible with the conclusion that the $14-$ to $16-\mathrm{kHz}$ peak is caused by drops in the 0.8 - to $1.1-\mathrm{mm}$ size range and the low-frequency sound by larger drops.

These results lead us to the main idea of this article: The 14- to $16-\mathrm{kHz}$ peak is a universal feature ${ }^{16}$ of rain noise and is produced by regular entrainment of bubbles. Our theory is supported by the fact that both the $14-$ to $16-\mathrm{kHz}$ peak and the regular entrainment process are surface tension dependent and vanish at the same value of the surface tension. Further evidence is provided by the pressure-time series of rain noise; these consist mainly of bubble oscillations. The theory does not agree with that of Nystuen, who explains the 14- to $16-\mathrm{kHz}$ peak in terms of initial impacts alone.

The apparent correlation between low-frequency sound and larger raindrops has not yet been thoroughly explained. Single-drop experiments show that large raindrops will produce much louder initial impact sounds than smaller ones because both their diameters and velocities are larger. They also seem to entrain much larger bubbles than those entrained by small drops. The low-frequency sound may be the result of a combination of these two effects. 


\section{CONCLUSION}

This work shows that the impact of a water drop can produce two distinct sounds: an initial impact and a bubble oscillation. It enables us to explain the $14-16-\mathrm{kHz}$ peak observed in the acoustic spectrum of rainfall on water in terms of regular entrainment of bubbles. The raindrops responsible for this process lie in a fairly small size range, but one that is common in most types of rain. The main evidence for this theory is that the spectrum contains bubble sounds and that the spectral peak can be removed by lowering the surface tension. The initial impact sound is probably not the cause of the $14-$ to $16-\mathrm{kHz}$ peak, but could possibly contribute to the general elevation of the noise level observed in heavy rain.

\section{ACKNOWLEDGMENTS}

The authors from the NCPA wish to acknowledge the financial support of the Office of Naval Research and the many helpful discussions with Andrea Prosperetti, who coordinates this project with the ONR.
'A. M. Worthington, Philos. Trans. R. Soc. London A189, 137-148 (1897).

${ }^{2}$ A. Mallock, Proc. R. Soc. London Ser. A. 95, 138-143 (1919).

${ }^{3} \mathrm{~W}$. Bragg, The World of Sound (Bell, London, 1920).

${ }^{4}$ M. Minnaert, Philos. Mag. 16, 235-248 (1933).

${ }^{5}$ G. J. Franz, J. Acoust. Soc. Am. 31, 1080-1096 (1959).

${ }^{6}$ T. E. Heindsman, R. H. Smith, and A. D. Arneson, J. Acoust. Soc. Am. 27, 378-379 (1955).

${ }^{7}$ N. Bom, J. Acoust. Soc. Am. 45, 150-156 (1969).

${ }^{8} J$. A. Scrimger, Nature 318, 647-649 (1985).

${ }^{9}$ J. A. Nystuen, J. Acoust. Soc. Am. 79, 972-982 (1986).

${ }^{10}$ J. A. Nystuen and D. M. Farmer, J. Acoust. Soc. Am. 82, 270-274 (1987).

"J. A. Scrimger, D. J. Evans, G. A. McBean, D. M. Farmer, and B. R. Kerman, J. Acoust. Soc. Am. 81, 79-86 (1987).

${ }^{12}$ J. E. Lane, "Microprocessor based rainfall distribution methods," University of Mississippi internal report (1987).

${ }^{13}$ R. Gunn and G. D. Kinzer, J. Meteorol. 6, 243-248 (1949).

${ }^{14}$ A. N. Dingle and Y. Lee, J. Appl. Meteorol. 11, 877-879 (1972).

${ }^{15}$ Y. Mori, M. Mizukami, and T. Bushimata, Jpn. Soc. Mech. Eng, 53, 894 902 (1987) (in Japanese).

${ }^{16}$ The idea that this peak was associated with some universal property of rainfall was first conceived by Andrea Prosperetti, who suggested that we look for some experimental explanation of the effect. 